\title{
DU PASSAGE DES SUBSTANCES MÉDICAMENTEUSES DANS LE LAIT
}

\author{
par Dr. J. KOLDA
}

Chef de travaux de l'Institut de pharmacologie de l'École vétérinaire de Brno (Tehécoslovaquie)

(Travail du laboratoire du Prof. Dr. O. Rувак, présenté à la Société de Biologie de Brno, 21 octobre 1925).

- FIN -

V. - Les autres substances organioues, exception faite des ALCALOÏDES.

10 Les déripés du phénol. - On n'a pas encore recherché directement le phénol et ses dérivés dans le lait, mais MAREK affirme que l'acide phénique, le bacillol, le lysol et la créoline passent dans le lait et lui prêtent son odeur caractéristique. Du fait que le lait 'prend l'odeur de ces substances, on ne peut pas conclure à leur passage dans le lait, puisque Domвrowsky a constaté que le lait prend très facilement l'odeur des dérivés du phénol quand il se trouve dans un lieu où on a manipulé ces derniers.

$2^{\circ}$ L'Urotropine (hexaméthylénetétramine). - Le passage de l'urotropine dans le lait avait été fréquemment recherché à cause de la grande valeur de ce médicament pour la désinfection interne. KoLDewiJn [5] décela par la méthode de Voisenet [165] le passage de l'urotropine dans le lait d'une vache qui en recevait $5 \mathrm{gr}$. pendant quatorze jours; elle éliminait 5 milligr. de ee médicament par le lait. Monvorsin administrait, en une fois, à une vache, 12 gr. d'urotropine. La réaction dans son lait était négative deux heures et demie après l'ingestion, mais elle était devenue positive dix heures plus tard; l'élimination était terminée au hout de vingt-cinq heures.

Bucura décela le passage de l'urotropine dans le lait des deux femmes auxquelles le médicament avait été administré à doses thérapeutiques. Rieder [166] arrive à la conclusion que la quantité d'urotropine éliminée par le lait est tout au plus $1 / 400$ de la quantité administrée; la plus grande concentration qu'il ait obtenue était de 1/40.000. Le passage de l'urotropine dans le lait de femme et de la chèvre a été également constaté par Vignes [167].

Le passage de l'urotropine dans le lait de femme et des animaux est certain, mais sa concentration thérapeutique peut difficilement être atteinte.

$3^{\circ}$ La phénolphtaleine. - Ce composé n'a pas été décelé par Bucura dans le lait des deux femmes qui en avaient reçu un demigramme par jour pendant deux jours. Koldewisn n'a pu le retrouver dans le lait d'une chèvre qui avait reçu pendant douze jours, au 
total, 11 gr. 5 de ce médicament. Van Italine a décelé la phénolphtaléine dans le lait d'une vache après l'administration par la bouche.

$4^{\circ}$ La fluorescéine. - V V In Italie décela le passage de ce colorant dans le lait de vache, mais KoLdewisn n'a pu le retrouver dans le lait d'une chèvre qui en recevait 1 gr. par jour pendant quinze jours.

La présenee des deux dernières substances dans le lait avait été suivie moins du point de vue toxicologique ou thérapeutique qu'en raison de leur facile passage dans les autres sécrétions. Puisqu'elles se laissent facilement déceler, on aurait voulu avoir, grâce à elles, un moyen qui permettrait de suivre leur traversée de la glande mammaire chez les différentes espèces et dans les divers états physiologiques ou pathologiques; mais les faits n'ont pas justifié ces espoirs.

$5^{\circ}$ L'huile de ricin. - Dolan a vu l'huile de ricin donné à des nourrices avoir une action purgative sur les enfants qu'elles allaitent. Selon Bollinger [168], les veaux sont tombés malades après que l'on a donné aux vaches les tourteaux de ricin. Eнrцich [169] ne pense pas que la ricine, qui est une toxalbumine, puisse s'éliminer par la glande mammaire ; il n'a pu obtenir une immunité durable chez les souris dont les mères avaient une immunité active contre la ricine.

$6^{\circ}$ Euphorbe. - House Lот [170] relate que des soldats, qui avaient fait usage de lait de chèvres ayant brouté l'Euphorbias paralias, furent pris de nausées, vomissements biliaires, diarrhée, refroidissement des extrémités, etc. Menzer [171] décrit aussi des intoxications chez des hommes qui ont pris le lait de chèvres ayant consommé de l'euphorbe.

Des autres médicaments organiques, Dolan ne pouvait pas constater le passage dans le lait pour l'huile de foie de morue et pour la scammonée. Après l'administration d'helminthol, Wesenberg [29] a obtenu une réaction à la formaline dans le lait.

Cronedi [162] et Bucura [6] ont recherché, avec un résultat négatif, le passage de la santonine dans le lait de femme; VAN Itallie [43] n'a pu, de son côté, la retrouver dans le lait de vache.

VI. - Les alcaloides et les glycosides.

Le passage des alcaloïdes dans le lait a toujours été suivi d'une attention particulière, parce que les alcaloïdes ne sont pas seulement des médicaments très fréquemment employés, mais ils se trouvent aussi dans différentes plantes qui peuvent être ingérées par nos femelles laitières. A cause de leur influence élective sur le système nerveux, ils deviennent très dangereux pour les enfants et les petits animaux qui parfois ont vis-à-vis d'eux une sensibilité particulière. Leurs réactions chimiques sont pour la plupart des réactions basées 
sur le changement ou sur l'apparition de eouleurs et elles ne sont authentiques que si les solutions sont plus ou moins pures. Dans la plupart des eas, surtout quand il s'agit de rechercher l'alealö̈de dans un mélange aussi complexe que le lait, leurs réactions biologiques ont beaucoup plus d'importance que leurs réactions chimiques.

10 L'opium et ces alcaloüdes. - Les alcaloüdes de l'opium sont de eeux qui sont décomposés dans leur passage à travers l'organisme. Parce qu'on ne pouvait pas retrouver la morphine dans l'urine, on a cru longtemps qu'elle se décompose dans le corps ; on sait aujourd'hui que la plus grande partie de la morphine, après l'injection souscutanée, s'élimine par la muqueuse gastrique et, en partie aussi, par la muqueuse intestinale. Seulement une petite quantité passe dans l'urine et une portion minime est retenue dans l'organisme. Pour l'élimination par le lait, il ne reste que des quantités infinitésimales, et pourtant, on trouve dans la littérature des observations cliniques sur la nocivité du lait des femmes qui ont reç de l'opium ou des injections de morphine.

Thовлнill [172] décrit un cas d'un nourrisson qui s'est endormi pendant quarante-trois heures après avoir pris le lait d'une nourrice qui a reç 20 gouttes de teinture d'opium. Hapton [173] a vu tomber malade un nourrisson âgé de sept mois qui a pris le lait d'une nourrice préalablement morphinisée. Evans [174] relate un cas de mort d'un enfant dont la mère a pris une grande dose de morphine. De même, Cŕrenvilue [175] a constaté des troubles très graves chez un enfant dont la mère avait pris une grande dose de morphine. Fubivi et Cantu [176] racontent qu'une femme qui avait pris du coton imbibé de laudanum dans une dent cariée, eut son nourrisson pris d'un coma qui dura plusieurs heures. Ils ont fait plus tard les expériences sur trois chiennes, auxquelles ils ont injecté 0 gr. 02 à 0 gr. 025 de morphine; la sécrétion du lait s'est abaissée en quelques jours de 50 cc. à 2 cc. La morphine n'a pu être trouvée dans le lait, mais par contre, ils ont constaté le passage de la morphine en traces seulement dans le lait d'une chèvre qui avait reçu les injections de celle-ci.

Les intoxications par le lait des mères morphinisées que nous avons eitées ci-dessus pourraient s'expliquer par la grande sensibilité des enfants vis-à-vis de la morphine. La plus grande dose qui puisse ètre employée chez eux est de 1 milligr. de morphine, e'està-dire deux gouttes de la teinture, mais encore au-dessous de cette dose, on a observé des accidents.

On a déerit aussi chez les animaux des intoxications par le lait qui devait contenir les alcaloïdes de l'opium. Wrecand [177] qui injecta à une jument, au cours de coliques, deux fois par jour, 0 gr. 4 de morphine, observa chez le poulain, après la tétée, l'indolence et 
la somnolence; le poulain s'est rétabli le deuxième jour. On comprend qu'une telle observation clinique n'est aucune preuve du passage de la morphine dans le lait, le lait des animaux atteints de coliques pouvant être lui-même noeif. Hannemann [178] observe que le lait devenait nocif pour les veaux si les vaches ont reçu du pavot (Papaser Rheas) avec leur nourriture verte; les veaux sont tombés, après la tétée, dans un état de prostration complète, même de paralysie, et quelques-uns ont succombé au cours de cette paralysie qui dura douze à vingt-quatre heures. Dès l'instant où l'on a cessé de donner du pavot avec la nourriture verte, les symptômes ont disparu. Cette observation n'est non plus une preuve du passage des alcaloïdes de l'opium dans le lait ; après administration de ladite nourriture verte, d'autres substances nocives provenant de celle-ei ont pu passer dans le lait.

Kuвıк [179], qui donne aux vaches et aux chèvres de grandes quantités du Papaser Rheas, n'a jamais constaté chez ceux-ci les symptômes de l'intoxication; leur lait n'avait aucune action nocive sur les animaux, sur les hommes et sur les enfants qui en ont pris.

A côté des simples observations cliniques, une série de travaux expérimentaux devait trancher la question du passage de la morphine dans le lait. Pinzani [180] a fait ses observations sur trente enfants dont les mères recevaient des doses thérapeutiques de morphine et il n'a jamais pu observer des troubles de la santé. Par une méthode analytique qui lui permettait de déceler encore 5 milligr. d'opium dans $100 \mathrm{cc}$. de lait, il n'a pu trouver aucune trace de morphine dans le lait. Bucura, également, n’a pas décelé la morphine dans le lait des femmes qui en recevaient des injections au moment de l'accouchement; il est vrai qu'aussitôt après l'accouchement, il ne pouvait avoir que très peu de lait pour l'analyse. LandsBERG [181] n'a pas trouvé de morphine dans le lait après l'injection sous-cutanée ou intraveineuse.

D'après ces expériences, on pourrait donc conclure que la morphine ne s'élimine point par le lait. Mais beaucoup plus convaincantes encore sont les expériences sur les animaux, avec lesquels on peut employer des doses très élevées. VAN ITallie [43] n'a pas constaté, par la méthode de Draggendorf, le passage de la morphine dans le lait d'une vache après une injection de 200 milligr.; il a ensuite administré pendant quatre jours $8 \mathrm{gr}$. d'opium pulvérisé à une vache et n'a pu non plus déceler la morphine dans le lait. KoldewisN recherche la morphine par la microméthode de BeHRENs dans le lait d'une chèvre qui a reçu chaque jour 250 milligr. de morphine sous la peau pendant douze jours ; jamais il n'a trouvé de morphine dans le lait.

Reiche [182] injecte à une chienne gravide, une à deux fois par 
jour, 4 à 50 milligr. de morphine, de façon que l'animal a reçu, en somme, au cours de vingt-cinq jours, $1 \mathrm{gr}$, de morphine. La chienne a mis bas des petits qui se sont portés très bien, malgré la répétition des injections de morphine à leur mère. La recherche de la morphine dans le lait avait été tout à fait négative, quoique la méthode employée fut très sensible et décelait encore 2 milligr. dans 100 cc. de lait. Les résultats de Lewald sur la recherche de la morphine dans le lait ont été également négatifs. FröHner [155] n'a pas eonstaté le passage de la morphine dans le lait, mais il a vu que la séerétion lactée diminue après ces injections, que le lait devient épais et coagule facilement. D'après Koвert [183] les substances dont l'odeur est caractéristique de l'opium passent dans le lait.

Möllmann [184] a fait des expériences sur les vaches et sur les chèvres, auxquelles il donnait l'opium per os et déterminait son passage dans le lait par la méthode chimique et par la méthode biologique sur les chiens. Une chèvre qui, pour Hess [185] est l'animal qui supporte le mieux l'opium parmi les animaux domestiques, a reçu pendant dix jours en somme 88 gr. d'opium; un agneau, allaité par cette chèvre, se portait toujours à merveille; l'analyse chimique par la méthode d'ERDMann a décelé une trace de la morphine dans le lait. Chez les autres animaux, vaches et chèvres qui ont reçu de grandes doses d'opium, le passage dans le lait par les deux méthodes ci-dessus n'a pu être constaté. Sauf dans un cas, chez une vache atteinte d'entérite, la morphine a été décelée par l'analyse chimique, mais la réaction physiologique est restée négative. L'administration d'opium abaisse la sécrétion lactée et la quantité de la graisse, mais le poids spécifique et le taux du lactose ne changent pas sous son influence.

Des autres alcaloïdes de l'opium, Bucura [6] n'a pas constaté le passage de la codéine et de la stypticine dans le lait de femme.

De toutes ces expériences, on peut conclure que l'opium et ses alcaloïdes ne passent pas dans le lait des fernmes et des femelles laitières après l'emploi des doses thérapeutiques. La quantité de morphine qui passa après l'administration de grandes doses dans le lait d'une chèvre et dans celui d'une vache atteinte d'entérite, était dans les expériences de Möllmann très petite et n'a pu être mise en évidence par l'administration du lait aux chiens ou aux agneaux. Relativement à l'élimination possible de la morphine par le lait, on rencontre une certaine divergence entre l'observation clinique et les résultats expérimentaux. Un jeune chien doit recevoir des doses au moins dix fois plus grandes pour présenter les signes d'intoxication qu'on reneontre chez l'enfant; dans le lait, il devrait done passer des quantités relativement grandes pour qu'elles puissent être décelées par l'épreuve biologique sur le chien. Les expériences 
de Möllmann qui ont été faites sur les ehiens ne peuvent pas servir de preuve absolument certaine de ee fait que le lait n'est pas nocif pour les enfants.

$2^{\circ}$ Atropine. - L'atropine peut s'éliminer dans le lait de femme, après son administration médicamenteuse, et chez les animaux après l'ingestion de la belladone. L'instinct alimentaire, qui est très engourdi chez les vaches, permet que les intoxieations par l'atropine soient possibles et, par suite ainsi, on a l'occasion de saisir le passage de l'atropine dans le lait. C'est surtout chez la chèvre qui est très peu sensible à l'atropine qu'il peut s'agir de son passage dans le lait sans que l'animal présente des signes d'intoxication. On ne possède pas une méthode chimique pour déceler le passage de l'atropine dans le lait et les expérimentateurs se sont servis uniquement des méthodes biologiques.

Fehling [24] donne à une nourrice de l'atropine à la dose de 1 à 6 milligr. et constate dans tous les cas la dilatation pupillaire chez le nourrisson. Il admet lui-même que ses observations ne sont pas une preuve pour le passage de l'atropine dans le lait, parce que les enfants après le sommeil ont toujours les pupilles dilatées. FubINI et BonAnNi [186] injectent à des chiennes et à des chattes de l'atropine à la dose de 1 milligr. Le lait pris après vingt-quatre heures a été injecté sous la peau de jeunes animaux qui ont tété les mères atropinisées; puisque le nerf vague des animaux tout jeunes n'a pas d'action inhibitrice sur le cœur, ils ont employé, pour les injections de lait, des animaux âgés au moins de vingt jours. Chez les animaux témoins, l'excitation du vague a déterminé le ralentissement du cœur, tandis que chez les animaux qui recevaient les injections du lait atropinisé, les excitations même le plus accentuées du vague n'ont pas déterminé l'arrêt du cœur. Comme, selon Rummo [187], il faut au moins 0 milligr. 25 d'atropine pour que son action sur le vague soit évidente, les auteurs conclurent alors que l'atropine s'élimine en proportion notable dans le lait.

Pour la recherche de l'atropine dans le lait, NAvratie [188] se servait d'une méthode biologique basée sur l'action de l'atropine sur le cœur de la grenouille. Cette action inhibitrice découle de la paralysie des centres cardiaques et elle se laisse facilement démontrer sur un cœur de grenouille lequel avait été auparavant arrêté dans son action par la muscarine; l'atropine écarte cette paralysie et met immédiatement le cœur en mouvement. Les cœurs des grenouilles ont été isolés et la circulation artificielle établie d'après la méthode de Hartung [189] modifiée convenablement par l'auteur pour son propre usage. Il a constaté au préalable l'activité de la muscarine (qu'il a préparée lui-même en partant de l'Amanita muscaria, et l'activité de l'atropine; le cœur arrêté par la plus petite 
quantité de muscarine peut être mis en mouvement par une dilution d'atropine de 1/14.000.000.

Les expériences ont été faites sur deux vaches, dont l'une a reçu une injection sous-cutanée de $0 \mathrm{gr}$. 1, l'autre seulement de $0 \mathrm{gr}$. 05 de sulfate d'atropine. Le lait était prélevé douze heures après l'injection. Le lait de la première vaehe mettait le çeur en mouvement à la concentration de $1 / 30$, celui de l'autre à la concentration de 1/20. II en découle que le lait concentré de la première vache contenait 0 gr. 0021, celui de l'autre 0 gr. 0015 d'atropine dans 1 litre. La dose maxima de sulfate d'atropine est de 0 gr. 001 pour l'enfant et de 0 gr. 003 pour l'homme. L'auteur déclare qu'un tel lait est nocif et recommande son exclusion dé la consommation.

$3^{\circ}$ La vératrine mérite une attention particulière à cause de sa grande toxicité. Pour l'homme, la vératrine l'est déjà à très petites doses; elle est fréquemment employée dans la pratique vétérinaire, notamment chez les vaches, sous la forme d'injections sous-cutanées. Dans le lait, la vératrine a été recherchée par Traxuer [190] par la méthode colorimétrique d'IPsen; l'auteur ne dit rien sur la sensibilité de sa méthode. Après administration d'une seule dose de $4 \mathrm{gr}$. de la racine d'ellébore blanc ou de 4 à $5 \mathrm{gr}$. de sa teinture à une chèvre, la vératrine a été recherchée dans le lait pendant les quatre jours qui ont suivi. Il n'a jamais décelé la vératrine dans le lait; dans l'urine, il n'en a trouvé que des traces vingt-quatre à trente heures après l'administration. L'auteur en conclut que le lait après l'administration des doses thérapeutiques de la vératrine peut être consommé sans aucun danger.

KAnNiK [3] employait pour la recherche de la vératrine dans le lait une méthode biologique très sensible, basée sur son action sur les muscles de la grenouille. Le gastrocnémien isolé de la grenouille se raccourcit après l'excitation électrique et revient ensuite à sa longueur normale; par la représentation graphique, on obtient une courbe qui est pour le même muscle, pendant un certain temps, constante. Si l'on badigeonne le muscle avec une solution de vératrine, la courbe de raccourcissement après l'excitation électrique change en ce sens que sa partie descendante est prolongée cinquante fois environ, le raccourcissement est plus prononcé et quand on emploie des solutions très étendues de vératrine, la courbe possède deux sommets. Le muscle avait été excité directement, car le nerf perd sa conductibilité après avoir été plongé dans le lait. La méthode a une sensibilité qui permet d'utiliser une solution aqueuse de vératrine à 1/1.000.000. Le lait des vaches qui possède une réaction neutre ou faiblement alcaline n'a aucune action sur la contractibilité du muscle. Mais Karnik constata que certaines substances du lait (qu'il appelle pour ce cas les substances de ballaste), empêchent la 
vératrine de développer son action spécifique sur le muscle. Après des recherches minutieuses, il est arrivé à débarrasser le lait de ces substances et il vit que le muscle donne la courbe caractéristique de la vératrine, quand celle-ci se trouve dans le lait dans une concentration de $1 / 100,000$.

Il injecta aux vaches, d'abord la dose thérapeutique ( 0 gr. 1$)$, ensuite la dose subtoxique $(0 \mathrm{gr}$. 15) de vératrine sous la peau et préleva le lait treize, vingt-trois, trente-cinq et quarante-cinq heures après l'injection. En aucun cas, la vératrine n'a pu être trouvée dans le lait par la méthode décrite plus haut. L'auteur en conclut que la vératrine ne s'élimine pas par le lait ou du moins que la quantité éliminée est plus petite que 10 milligr. dans 1 litre, ce qui ne peut pas déterminer aucun trouble chez l'enfant ou chez les animaux à la mamelle.

$4^{\circ}$ La cytisine. - Si cette substance s'éliminait par le lait, elle serait selon Ковегт [183], très dangereuse, parce que les chèvres, qui mangent très volontairement les différentes sortes de Cytisus, supportent de grandes quantités d'alcaloïde, sans présenter des signes d'intoxication. Radzrwillowicz [191] constata que la dose mortelle pour le chien est dè 2 à 3 milligr. et pour la chèvre, 73 milligr. ; d'après lui, les ehèvres éliminent la cytisine par le lait sans présenter aucun signe d'intoxication. Selon Rosenthal [192], on avait constaté fréquemment en Dalmatie, où les chèvres mangent le Cytisus Weldeni, des intoxications par leur lait chez l'hommme.

KoldewiJn [5] employait pour la recherche de la eytisine la méthode de VAN DEs Moer [193] qui décèle encore 2 milligr. 5 de cet alcaloïde dans 100 cc. de lait. Une, chèvre recevait 3 à 100 gr. de graines de Cytisus Liburnum qui contiennent environ $1 \%$ de eytisine. Malgré l'administration prolongée pendant trois semaines, il n'a jamais décelé dans le lait la cytisine. Un petit ehat nourri par le lait de cette chèvre, n'a présenté aucun signe d'intoxication.

Au point de vue du passage de la cytisine dans le lait, les résultats des auteurs sont contradictoires et les recherches poursuivies en ce sens à l'Institut pharmacologique de l'Ecole vétérinaire de Brno doivent résoudre cette question, nous l'espérons.

$5^{\circ}$ L'yohimbine. - Selon Holterвach [194], la yohimbine augmente la sécrétion lactée et le poids spécifique du lait sécrété. Son élimination par le lait a été suivie par Topol [195] qui a donné la yohimbine de SPIEger à une chèvre pendant dix-neuf jours à la dose de 0 gr. 03 par jour. Dans le lait et dans l'urine, la yohimbine a été recherchée pendant toute la période de l'administration et encore six jours après que celle-ci avait pris fir. Elle n'a jamais été trouvée dans le lait, mais dans l'urine, elle apparaissait six à dix-huit heures après l'administration. Le poids spécifique du lait augmentait 
déjà après la première dose et il est revenu à son taux normal après la fin de l'administration.

$6^{\circ}$ La colchicine. - De même que la cytisine, les chèvres supportent aussi de fortes doses de colchicine, sans présenter des signes d'intoxication (RAtri [196]). Le lait des vaches qui ont mangé du colchique était toxique même dans les cas où on n'a pas constaté de trouble chez elles. Housenot [170] relate que les habitants d'un quartier à Rome furent atteints de diarrhée, vomissements, soif intense, abaissement du pouls et de la température. Les médecins ont soupconné le lait de chèvre qui est d'un usage général dans ce pays. Les animaux eux-mêmes se sont montrés à l'examen sains, mais dans leur lait et dans les vomissements des malades on a trouvé les réactions propres à la colchicine.

70 La quinine. - Dolan, Chevalier et Henry n'ont pu démontrer le passage de la quinine dans le Jait. Bucura a donné à deux femmes jusqu'à 0 gr. 4 de quinine sans pouvoir le déceler dans le lait.

LEWALD prétend que le sulfate de quinine s'élimine très facilement par la mamelle et qu'on peut guérir la fièvre intermittente du nourrisson en administrant le médicament à la nourrice. Selon Our [197] le sulfate de quinine administré à la dose de 75 centigr. à 1 gr. se retrouve dans le lait en très petites quantités; l'analyse quantitative n'a pu être faite qu'une seule fois et sur 100 cc. de lait dans lesquels il fut trouvé 2 à 3 milligr. de quinine, ce qui est une quantité si petite qu'elle ne peut pas altérer la santé du nourrisson. La quinine apparaît dans le lait deux à trois heures après son administration. La conclusion de Koldewion [5] est semblable à celle de Our: La quinine passe en très petites quantités dans le lait de vache.

LEWIN [144] en parlant de l'élimination de la quinine par le lait fait remarquer qu'il y a déjà plus de cent ans qu'on donne à boire aux nourrissons atteints de la fièvre intermittente le lait des nourrices auxquelles on a administré de la quinine. Burder [198] constate que la quinine administrée à jeun s'élimine très vite par le lait en quantité telle, qu'elle peut avoir une action toxique sur le nourrisson.

Parmi les autres adcaloïdes, la nicotine doit s'éliminer, d'après Ковевт [183], par le lait; selon Houselot [170] et G. Lyon [199], le lait des femmes employées dans les manufactures de tabac, provoque chez leurs nourrissons des coliques et même des accidents nerveux.

Le passage de l'hydrastine dans le lait n'a pas pu-être démontré par Bucura. Hammerbacher [200] a fait une série d'expériences sur l'influence de la pilocarpine sur la sécrétion lactée et il en conclut 
que cet alcaloïde n'a aucune action galactogogue, mais qu'il diminue au contraire la sécrétion lactée ; VAN ITALLIE [13] n'a pas constaté son passage dans le lait après l'injection sous-cutanée. Les recherches de la pilocarpine dans le lait faites par StumpF [33] étaient également négatives.

La physostigmine n'a pas été retrouvée dans le lait par VAN ITALLiE.

Dolan a fait prendre de la eiguë et ne parvint pas à retrouver dans le lait de la coniine. Le même auteur fit prendre à une femme 11 gouttes de teinture d'aconit jusqu'à 12 gouttes ; il n'a pu retrouver l'aconitine dans le lait et ne remarqua aucun trouble dans la santé de l'enfant. Sonnenberg [201] croit à l'élimination de l'aconit par le lait. Dolan a abouti à un résultat négatif en ce qui concerne le passage de l'ergot de seigle dans le lait. Selon Monvorsin [44] la strychnine doit s'éliminer en petite quantité par le lait.

Au point de vue du passage dans le lait, la solanine est redoutable. Les femelles laitières peuvent la recevoir avec les drêches, les pommes de terre crues et sur le pâturage avec les nombreuses solanácées qui en contiennent. Les vaches sont peu sensibles à la solanine, de façon qu'il pourrait y avoir une élimination par le lait, sans qu'aucun trouble de la santé de l'animal sécréteur fut constaté.

C'est en se basant sur ces considérations que ZeMLJIC [202] a recherché le passage de la solanine dans le lait au moyen d'une méthode biologique basée sur l'action hémolytique et trypanolytique de ce poison. Il constata tout d'abord que le lait des vaches, quoique sa pression osmotique est autre que celle du sang, n'exerce aucune action hémolytique sur les globules rouges du mouton. La méthorle hémolytique permet de déceler la solanine à la concentration de 1/2.000; en sensibilisant cette méthode, il est arrivé à déceler la solanịne à une concentration de $1 / 5.000$, e'est-à-dire $0 \mathrm{gr} .2$ dans 1 litre. L'action trypanolytique de la solanine avait été étudiée sur les trypanosomes de la dourine; cette action peut encore très bien être observée à une concentration de la solanine de $1 / 21.000$, c'està-dire qu'on peut déceler par cette méthode 0 gr. 0047 de solanine dans 1 litre de lait.

Après avoir fait aux vaches des injections sous-cutanées de la solanine à la dose de $0 \mathrm{gr} .5$ pour $1 \mathrm{kgr}$. de poids vif, il n'a pu déceler celle-ci dans le lait prélevé douze et vingt=quatre heures après l'injection. Il en conclut à la non-élimination de la solanine par le lait; mème si la solanine s'élimine par le lait ce sera toujours en une quantité plus petite que 0 gr. 0047 par litre.

Le passage des glucosides de la digitale dans le lait a été recherché par Dolan sans succès. 


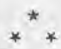

Parmi les médicaments organiques, ceux dont a le mieux étudié le passage dans le lait sont, sans nul doute, les anesthétiques du groupe de l'alcool. Grâce à la technique excellente de M. Nrccoux, le passage de l'alcool, de l'éther et du chloroforme est certain, mais la même technique nous a permis de constater que, ici encore, les quantités des substances ingérées sont formidables vis-à-vis de celles qui ont été retrouvées dans le lait; la nocivité du lait des femmes soumises à une anesthésie générale par l'éther ou le chloroforme est très réduite. Puisque l'alcool passe aussi dans/le lait en très petites quantités, il faut ehercher la cause de la nocivité du lait des femmes adonnées à l'alcoolisme et des femelles Iaitières qui reçoivent des drêches dans l'élimination de substances nocives autres que l'alcool.

Parmi les acides organiques, c'est l'acide salicylique qui passe le mieux dans le lait après l'administration par la bouche, mais e'est toujours en petites quantités. La teneur du lait en acide citrique qui est un composé normal du lait ne peut être augmentée par une administration expérimentale.

L'élimination des purgatifs anthraquinoniques n'est pas encore certainement démontrée. La plupart des essences s'éliminent aussi par le lait; puisque les essences se trouvent dans certaines boissons alcooliques, la nocivité du lait des femmes adonnées à l'alcoolisme peut très probablement être rattachée à l'élimination des essences par cette sécrétion.

En ce qui concerne le passage des alcaloïdes dans le lait, l'observation clinique peut à peine résoudre cette question au point de vue quantitatif. Seule, la méthode chimique et mieux encore la méthode biologique, nous renseignent de plus près sur les quantités des alcaloüdes qui passent dans le lait. Par deux méthodes biologiques différentes, on a démontré que l'atropine peut passer dans le lait en quantités telles qu'il peut provoquer une action nocive chez le nourrisson. Des autres poisons parasympathiques, le passage de la scopolamine a été décelé dans le lait, tandis que celui de l'hyoscine, de la physostigmine et de la pilocarpine n'a pas encore été démontré. La vératrine et l'yohimbine ne s'éliminent pas par le lait, même après l'emploi des doses subtoxiques; avec la cytisine, les résultats obtenus jusqu'à présent ne sont pas univoques. Les alcaloïdes de l'opium ne s'éliminent pas dans le lait des femmes et dans celui des femelles laitières saines après l'administration des doses thérapeutiques; même après l'administration de doses très élevées et chez les individus malades, l'élimination par le lait se fait en si petites quantités que la santé des nourrissons n’en peut pas être incommodée. 
La non-élimination de la morphine par la glande mammaire se laisse facilement expliquer par l'élimination relativement rapide et importante par la muqueuse gastrique.

Par le lait s'élimine encore la nicotine, la colchicine et la quinine ; avec ce dernier, l'élimination est cependant si minime qu'il ne peut être question de l'emploi thérapeutique d'un tel lait. La solanine ne s'élimine pas dans le lait en quantités décelables par l'analyse et la nocivité des vaches nourries de drêches doit avoir une autre cause que le passage de la solanine dans le lait.

Comme on le voit, les travaux qui ont porté sur le passage des médicaments dans le lait sont nombreux. La conclusion la plus importante que l'on en peut tirer est que la plupart des médicaments s'éliminent dans le lait. Les résultats négatifs que certains expérimentateurs ont obtenus peuvent trouver leur explication dans une ou plusieurs des causes suivantes :

a) On ne connaissait pas bien tous les composants normaux du lait : l'acide citrique, le lithium, le cuivre, ce qui a causé certaines erreurs dans la détermination quantitative de leur passage dans le lait.

b) Beaucoup d'erreurs sont causées par une technique chimique ou biologique défectueuse. Au fur et à mesure que les méthodes ont été perfectionnées et rendues plus sensibles, les résultats négatifs sont allés en diminuant. L'alcool ne pouvait pas être décelé dans le lait des femmes par le pycnomètre, le vaporimètre ou par l'épreuve à l'iodoforme; en employant la méthode sensible et précise de Nicloux, cet auteur et plusieurs autres après lui ont alors décelé l'alcool dans le lait des femmes qui avaient reçu des doses plús petites même qu'auparavant. D'un autre côté, les méthodes sensibles ont permis ultérieurement d'expliquer certaines erreurs de méthodes insuffisantes, utilisées au début, comme c'est, par exemple, le cas pour la rhubarbe.

c) Une faute très fréquente est l'emploi de doses trop petites pour la recherche du passage des médicaments dans le lait. Nous trouvons un exemple de l'importance de la dose employée dans les recherches sur le passage de l'iode dans le lait; avec une seule dose de 10 gr. d'iodure de potassium, l'iode peut être décelé dans le lait, tandis qu'avec 3 gr., l'épreuve est négative. Les résultats négatifs de Van Itallie sur l'élimination de la morphine deviennent positifs avec Neuman qui a élevé les doses. La concentration des anesthétique généraux, éther et chloroforme, éliminés par le lait, augmente au fur et à mesure que la dose administrée s'élève.

d) Enfin la dernière cause d'erreur de certains auteurs est la 
clôture précoce de leurs recherches. Le meilleur exemple nous est fourni par les expériences de Sigalas et Dupouy et celles de Louïse et Moutrer avec le mercure; l'élimination de celui-ci par le lait chez la ehèvre ne commence que le dixième jour au cours d'une administration journalière des sels de mercure. Ce retard dans l'élimination du mercure par le lait explique les résultats négatifs obtenus par Dolan, Kahler, HaAs, Somma et d'autres. L'élimination du plomb par le lait se fait aussi avec un retard prononcé.

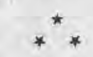

Nous avons bien vu que la plupart des médicaments passe dans le lait; mais, en tous cas, il faut ajouter que l'élimination se fait toujours en très petites quantités. Même après l'administration de doses très élevées, données pendant un temps prolongé, l'élimination ne se fait que rarement en une quantité telle qu'elle puisse se prêter à une détermination exacte. Aussi, peut-on adopter aujourd'hui intégralement la formule énoncée en 1909 par M. Porcher, dans son rapport à la Commission du lait de la Ligue contre la mortalité infantile : « Lorsque la mamelle élimine certaines substances qui ont été administrées thérapeutiquement ou expérimentalement, cette élimination est des plus réduites et il faut faire prendre à l'animal en expérience une quantité vraiment considérable du corps à rechercher pour n'en saisir dans le lait que des traces ou si l'on préfère une proportion vraiment infime par rapport à ce qui a été administré. "

Puisque l'élimination des médicaments par le lait ne se fait, en général, qu'après l'administration de doses élepées pendant un temps prolongé, on peut examiner la valeur de l'opportunité des règlements locaux de certaines villes allemandes contre la vente du lait des vaches soumises au traitement par certains médicaments. La médieation peut être continue ou momentanée; dans le dernier eas, il est très probable que l'agent thérapeutique ne passera pas dans le lait ou du moins que ce passage sera tellement réduit qu'il n'y aura pas lieu de craindre l'action nociye sur le nourrisson. Le traitement continu se fera, soit au cours des maladies aiguës pendant lesquelles la sécrétion lactée se tarit très vite, soit au cours des maladies chroniques qui dépriment tellement l'organisme, que l'exploitation de la femelle laitière n'est plus rémunératrice. En tous cas, ces anciens règlements ne répondent plus aux connaissances nouvelles sur l'élimination des médicaments par le lait.

On y trouve l'interdiction de la vente du lait de vaches qui ont reçu de la vératrine et de l'opium; or, les recherches chimiques et. biologiques ont suffisamment démontré que la vératrine ne passe pas dans le lait, même après l'administration de doses très élevées ; 
les alcaloïdes de l'opium ne passent non plus dans le lait après l'administration des doses thérapeutiques. Ces règlements ne disent rien de l'atropine employée aujourd'hui de plus en plus dans la thérapeutique vétérinaire et qui, seule entre les alcaloïdes, peut passer dans le lait en quantité nocive. L'interdiction d'utiliser le lait des vaches soumises au traitement par les mercuriaux n'est pas motivée ; l'hypersensibilité des bovidés vis-à-vis d'eux est bien connue et ne permet que l'emploi de doses très petites ; et les faits expérimentaux nous montrent l'élimination du mercure par le lait après l'administration prolongée de grandes doses, se fait en si petite quantité, que c'est diffieilement que l'on entrevoit la possibilité de s'en servir dans un but thérapeutique.

\section{Bibliographie}

[1] Porcher, Rapport à la Commission du lait de la Ligue contre la mortalité infantile, l'Hygiène de la viande et du lait, p. 425, 1909,

[2] Déchavanne, Du passage des substances médicamenteuses et toxiques dans le lait, Thèse de Lyon, 1909.

[3] Karnik (L.), Etude sur l'élimination de la vératrine dans le lait et recherche par la méthode biologique, Thèse de Brno, 1922.

[4] Gérard et Meurin, Bull. Soc. Chim., 1908.

[5] Koldewijn, Arch. de Pharm., XLVIII, p. 623, 1910.

[6] Bucura, Zs. f. exp. Path. u. Therap., IV, 398, 1907.

[7] Chevalier et Henry, Mém. sur le Lait, Paris, 1839.

[8] Péligot, Ann. de Chim., LXII, p. 422, 1836.

[9] Leviseur, Journ. f. Kinderkrankh., p. 373, 1872.

[10] Jensen, Annuaire agricole de la Suisse, 1905, Res. Gén. du Lait, IV, p. 276, 1905.

[11] PAGÈs, l'Hygiène des animaux domestiques dans la produciion du lait.

[12] Schutte-Brauninghaus, Molk. Ztg., p. 601, 1903.

[13] Fuchs, Cité d'après no 22.

[14] Cité d'après Henseval et Muluie, no 22.

[15] Cité d'après Henseval et Mullie, no 22.

[16] Sснгорт, Jahresber d. mlichw. Versuchstation zu Kiel, p. 8, 18841885.

[17] Bodné, Cité d'après Henseval et Muluie, no 22.

[18] Broquet, Cité d'après Henseval et Mullie, no 22.

[19] Szilasy, Repertor. d. analyt. chem., p. 436.

[20] Mosslinger, Ber. ueber. d. VII Versammlung. Bayer. chem. i. Speyer, 1888.

[21] Fritzmann, Zs. f. oeff. Chem., p. 610, 1897.

[22] Henseval et Mullie, Rev. Gén. du Lait, 1905.

[23] Dolan, The practitioner, XXVI et XXVII, 1881.

[24] Fehring, Arch. f. Gynaekol, XXVII.

[25] LAXA, le Lait, II, p. 428, 1922.

[26] Zuckmayer, Pfluegers Arch., CLVIII, p. 209, 1914.

[27] Loughin, Répertoire de Pharmacie, p. 551, 1873. 
[28] Rosenhaupt, Arch. f. Kinderheik, XL. p. 131, 1904.

[29] Wesenberg, $Z s$ f. angesp. Chem., XXIII, 1347, 1910.

[30] Schlossberger; Wurtemberg. Korrespondenzblat, no 20, 1851.

[31] Schauerstein et Spaeth, Jahresber. $f$. Kinderheilk, II, p. 13, 1858.

[32] Schafer, Zs f. wien. Aertzte, no 5, 1859.

[33] Stumpf, Arch. f. Klin. Med., XXX, nos 2-3, 1882.

[34] Harnier, Thèse de Marburg, 1847.

[35] Welander, Nord. med. Arch., 1874.

[36] Wallace, Cité d'après Déchavanne; no 2, p. 22.

[37] Whaler et Herberger, Wagner's Handspoerterbuch d. Physiol., Milch.

[38] Lazansky, Vierteljahrschrift f. Dermatol, u. Syphilis, p. 43, 1878.

[39] Flamini, Reoue mensuelle des maladies de première enfance, 1902.

[40] Reisst-Scheffer (M.), Arch. der Pharm., p. 246-595, 1908.

[41] Simon, Die Frauenmilch nach ihrem chemisch-physiol. Verhalten, Berlin, 1838.

[42] Labourdette et Dumesnil, Gaz. des Hôp., p. 223, 1856.

[43] Van Itallie, Pharm. Weekblad, XLI, p. 506, 1904 et Tijdschrifi poor Veeartsenijkunde, p. 479, 1904.

[44] Monvorsin, le Lait, Paris, 1920.

[45] Loens, Berl. kl. Wochschr., p. 2064, 1911.

[46] Mueller, Fuehling's landw. Ztg., LII, p. 630.

[47] Caspari, Arch. f. Physiol. , Supplement band, p. 267, 1899.

[48] Winternitz, Deutsch. med. Wochenschr., no $30,1897$.

[49] Engel, Chem. Ztg., no 27, 1905.

[50] Siegfeld, Milchsirtsch. Zentralbl., I, p. 244, 1905.

[51] Gogrtidse, Zs. f. Biol., XLV, p. 353, 1904 et XLVI, 1905.

[52] Pflueger, Pflueger's Arch., LXXXV, 1, 1901.

[53] Jantzen, Centralbl. f. Physiol., XV, p. 508, 1901.

[54] Morat et Doyon, Traité de Physiologie, t. I, p. 404.

[55] Mossé et Cithala, Acad. de Méd., IV, 2, 1898 et Semaine Méd., XVIII, 171.

[56] Bang, Berl. klin. Wochschr., p. 52, 1897.

[57] Bramweld, The Lancet, 1890.

[58] Gemmex, Berl. klin. Woch., no 15, 1877.

[59] Sanson, C. R. de la Soc. de Biol., p. 154, 1894.

[60] De Reurl, Cité d'après Karnik, no 3.

[61] Neumann, Milchzeitung, XXII, p. 141.

[62] Golding et Paine, The Analyst, XXXV, p. 246, 1910.

[63] Jondan, Amer Journ. of. Physiol, XVI, p. 268.

[64] Fingerling, Bioch, Zs., XXXIX, p. 239.

[65] Brouardel et Pouchet, Annales d'Hyg. publique et de Méd. légale, p. $73,1881$.

[66] Hentwig, Untersuchungen ueber den Uebergang und das Verweilen des Arseniks in dem Tierkorper, Berlin, 1847.

[67] Spallanzani et Zappa, Cités d'après Froehner, Toxicologie.

[68] Labourdette, Bull. Acad. de Méd., XXIV, p. 746, 1858-1859. 
[69] Bloemendal, Arsenicum in het dierlijk organisme, Thèse, Leiden, 1908.

[70] Lewald, Untersuchungen ueber den Uebergang pon Arzeneimitteln in die Milch., Breslau, 1857.

[71] Ewald, Berl. klin. Woch., p. 554, 1885.

[72] Jessionex, Muench. med. Woch., no 22, 1911.

[73] Adele et Arhur Bornstein, Deutsche med. Woch., XXXVII, p. 1120, 1911 .

[74] Caffarena, la Pediatria, XX, p. 245, 1912.

[75] Koeller, Ueber die Ausscheidung von Arsen durch, die Milch, Thèse, Hanover, 1912.

[76] TAEGE, Muench. med. Woch, no 33, 1910.

[77] Duнот, même périodique, $\mathbf{n}^{0} 35,1910$.

[78] Chamberlain et Chevrier, C. R. de la Soc. de Biol., CXXI, 136, 1911.

[79] Uhlenhuth et Mulzer, Deutsche med. Woch., no 19, 1913.

[80] Harms, Hannover's Jahresber., IV.

[81] Kerbasch, Arch. den Pharm., p. 246, 1908.

[82] Kahlen, Vierteljahrschrift f. pralet. Heilk., 1875.

[83] Сомма, la Pediatria, XIX, p. 168, 1899.

[84] HaAs, Cité d'après Raudnitz, Sammelreferat ueber die Arbeiten aus der Milchchemie, Leipzig.

[85] Ludwig, Zs. f. anal. Chem., XX, 1881.

[86] Orfila, Cité d'après Déchavanne, no 2.

[87] Louïse et Moutier, C. R. de la Soc. de Biol., I, p. 878, 1906.

[88] Mauriac, Traitement de Syphilis, p. 723.

[89] Kunn, Viertelj. f. Dermatol. u. Syphilis, n 2, 1876.

[90] Vajda et Paschis, Ueber den Einfluss des Quecksilbers auf den Syphilisprocess, Vienne, 1890.

[91] Sigalas et Dupouy, Congrès International de Méd. Path. Gén., p. 394, Paris, 1900.

[92] Cullenier, Bull. de Thérap., t. XLII, p. 437, 1852.

[93] Baum et Sefelger, Berl. Arch., 1898.

[94] Bahrdt et Edelstein, Zs. $f$. Kinderheilk., I, p. 182, 1910.

[95] Liebreich, cité d'après Nothnagel et Rossbach, Arzeneimittellehre, 1894.

[96] Staffond, Zs. $t$. Kinderheilk, IV, p. 168, 1912.

[97] Bistrow, Virchov's Arch., XLV, p. 98, 1869.

[98] Giondani, Reץ. des Maladies de l'enfance, 1902.

[99] Sохнцет, Versammlung des deutsch. Vereins f. oeff. Gesuncheitspflege $z u$, Leipzig, 1891.

[100] Herpes, Untersuchungen ueber den Eisengehalt der Milch., Thèse, Hanover, 1914.

[101] Nоттвонг et Doerr, Zs. f. Nahrungs $u$. Genussmittel, XXVIII, p. 417, 1914.

[102] Kunkel, Toxikologie, I, p. 72, 1898.

[103] Lindner, Biedermann's agrikulturchem. Zentralbl., XXXIII, p. 70.

[104] Wedemann et Trtze, Arb, a. d. kaiserl. Gesundheitsamte, XXXVIII, p. 125, 1911. 
[105] Масн, Cité d'après Tschirch, Das Kupfer pom Standpunkte der gerichtl. Chemie, Stuttgart, 1893.

[106] Hess, Supplee et Beluis, Journ. of. Biol. Chem., LVII, p. 725, 1923.

[107] Rost, Arch. internat. de Pharm. et de Thérap., XV, p. 291, 1905.

[108] Willey, Journ. of Biol. chem., III, p. 11.

[109] Sснмірт, Journ. f. Landss., XXVI; 405, 1878.

[110] Vernay, Lyon Méd., IV, 440, 1872.

[111] Soltmann, Handbuch d. Kinderkrankh. von Gerhardt, V, p. 45, 1880.

[112] Meunier, Journ. de Méd. et de Chirurg. prat., p. 293, 1898.

[113] Сомве, même périodique, p. 420, 1898.

[114] BaUm et Illner, Volkmann's klinische Vortrage, no 105, 1894.

[115] Kingemann, Virchow's Arch., p. 72, 1891.

[116] Rosemann, Arch. $f$. d. ges. Physiol., LXXVIII, p. 52, 1899.

[117] Nicloux, C. R. de la Soc. de Biol., L., p. 295 ; Obstétrique, p. 97, 1900 ; Recherches expérimentales sur l'élimination de l'alcool de l'organisme, Paris, 1900.

[118] Olow, Bioch. Zs., CXXXVI, p. 553, 1923.

[11.9] Voeltz et Paechtner, Bioch, Zs., LII, p. 73, 1913.

[120] Voeltz, Baudrexel et Dietrich, Arch. f. d. ges. Physiol., 1910 1912.

[121] Duennwald, Ueber Alkoholauscheidung durch die Milchdruese unter bes. Beruecksichtigung der Gesvoehnung, Thèse de Hannover, 1919.

[122] Wever, Ueber die Wirkung pon Aethylalkohol auf die Laktation von Ziegen, Thèse de Hannover, 1919.

[123] Girard, Soc. de Méd. publique, IV, 23, 1884.

[124] Toussaint, Revue d'Hyg., p. 932, 1885.

[125] Dемме, Ueber den Einfluss des Alkohols auf den Organismus des Kindes., Stuttgart, 1891.

[126] Arnould, Cité d'après Rouvier, le Lait, Paris, 1893.

[127] Rosкam, Ann. de la Soc. Méd.-Chirur. de Liège, 1895.

[128] Périer, Ann. de Méd. et de Chir. infantile, p. 479, 1898.

[129] Toulouse, Gazette des Hôpitaux, p. 914, 1891.

[130] Teichert, Milchztg., no 10, 1901.

[131] Marfan, Traité de l'Allaitement, Paris, 1893.

[132] Gorup et Besanez, Arch. $f$. Phys. Heilk., VIII.

[133] Nrcloux, C. R. de la Soc. de Biol., I, p. 347, 1908.

[134] Gramén, Acta Chirurg. skandinas.., Supplement bd., 1, 1922.

[135] Nicloux, C. R. de la Soc. de Biol., I, p. 720, 1906.

[136] Scanzoni, Beitr. zur Geburtskunde, II.

[137] Sтовсн, Berl, tier. Woch., p. 316, 1902.

[138] Richter, Charité Ann., III, 1878.

[139] Hoerder et Herdergen, Arch. f. Gynaekol., X.

[140] Paulr, Uebergang der Salicylsaure in die Milch der Wochnerinnen, Thèse de Berlin, 1879.

[141] Pinzani, Ann. di Chim. e di Farm., XIII, p. 253.

[142] Guibert, Archives de Toxicologe et de Gynécologie, 1891.

[143] Fieux, Revue internat, de Méd. et de Chirurg. prat, , no 18, 1887,. 
[144] Lewin, Die Nebensvirkungen d. Arzeneimittel, 1893.

[145] Stewenson, Journal of the Mitchigan State Med. Soc., XIII, p. 230 1914.

[146] Sохнцет, Zs. d. landw. Vereins in Bayern, p. 37, 1888.

[147] Vaudin, Cité d'après Oppenheimer, Handb. d. Bioch., III, 1, p. 395.

[148] Soeldner, cité d'après Oppenhermer, Handb. d. Bioch., III, 1, p. 395 .

[149] Scheibe, cité d'après Sommerfeld, Handb. d. Milchkunde, p. 803.

[150] Gilibenti, Delle latte degli animali alimenteli con Oxalis Cernua e dell'influenca di questa sull'organismo animale, Palermo, 1888.

[151] Bregnius, Cité d'après Dombrowsky, n⿳0 152.

[152] Dомвпоwsкy, Arch. f. Hyg., L, p. 183, 1905.

[153] Marchetti, Maly's Jahresber, p. 266, 1897.

[154] Uмгост, Jahresber. $f$. Kinderheilk., XLII, p. 356, 1896.

[155] Froenner, Lehrbuch d. Arzeneimittellehre f. Tieraertzte, 1919.

[156] Choetta-Filehne, Lehrbuch d. Arzeneimittellehre, 1896.

[157] Gotтlieb-Meyen, Experimentelle Pharmakologie, p. 227, 1922.

[158] Heins, Arzeneimittellehre, p. 214, 1907.

[159] Grimmer, Cité d'après Grimm, Chemie u. Physiologie der Milch.

[160] Ayers et Johnson, Jahresber. ueber die Fortschritte d. ges. Med Vet., XXXIV, p. 262, 1916.

[161] Connevin, C. R. de la Soc. de Biol., CXLVI, p. 263.

[162] Cononedi, Maly's Jahresber. f. Tierchemie, p. 178, 1895.

[163] Mareк, Lehrbuch d. klin. Diag. d. int. Krankh. d. Haustiere, 1912.

[164] Hess, Schaffer et Bodzynski, Landw. Jahresber. d. Schrveiz., II, p. $56,1890$.

[165] Vorsenet, Bull. Soc. Chim. de Paris, XXXIII.

[166] Rieder, Monatschr. f. Kinderheilk, XI, p. 80, 1912.

[167] Vignes, Soc. d'Obstétrique et de Gynécol. de Paris, 1912.

[168] Bollinger, Deutsch. Zs. f. Tiermed., VI.

[169] Енrlich, Zs. f. Hyg., XII.

[170] Houselot, Cité d'après Déchavanne, no 2.

[171] Menzer, Cité d'après Froenner, Toxikologie, s. 261.

[172] Thonnhill, Cité d'après Marchand, Lehrbuch der physiologishen Chemie.

[173] Harton, Arch. f. animal. Nahrungsmittelkunde, VI, s. 25.

[174] Ewans, British Med. Journ., II, p. 1159, 1885.

[175] Cérenville, Réf. chez Gibson, Cheyne-Stokes Resp., p. 30, Edinburg, 1892.

[176] Fuвini et Cantu, Moleschots Untersuchungen zur Naturlehre, 1892 , IV, 396.

[177] Wieland, Berl. tier. Woch., p. 793, 1905.

[178] Hannemann, Preuss., Vet. Ber., 1904.

[179] Kuвıк, les Expériences sur la toxicité du Papaser Rhoecis, Thèse de Brno, 1924.

[180] Pinzani, Gazette degli Ospit., 1889.

[181] Cité d'après Ennst, Grundriss d. Hyg. f. Tierarzte, p. 157, 1913. 
[182] Reiche, Ueber den Einfluss einer trachtigen Hundin gegebenen Morfumgaden auf den Hundefoetus und saugenden Hund., 1912.

[183] Ковект, Lehbuch der Intoxikationen, II, p. 1176, 1906.

[184] Moellmann, Untersuchungen ueber den Uebergang von Opiumal. kaloiden in die Milch unserer Haustiere, Thèse de Zurich, 1910.

[185] Hess, Thèse de Berne, 1901.

[186] Fubini et Bonanni, Ann. di Chim. e di Farm. XIII, p. 253.

[187] Rummo, Congrès de Méd. Int., Rome, 1899.

[188] Navratr, De l'élimination de l'atropine par le lait, Thèse de Bmo, 1924.

[189] Hartung, Arch. f. exp. Pathol. u. Pharm., LXIX, p. 149.

[190] Traxler, Zs. f. Fleisch. u. Milchhyg., XXXII, p. 18.

[191]. Radziwillowicz, Arb. a. d. Pharm. Inst. zu Dorpat, II, p. 56, 1888.

[192] Rosenthal, Cité d'après Koldewijn, no 5.

[193] VAN der Moer, Thèse de Groningen, 1890.

[194] HostenвAсh, Tieraerztl, Rundschau, no 2, 1902.

[195] Topol, Z. f. Fleisch. u. Milchhyg., XXXI, p. 71, 1920.

[196] Ratti, Ungar. pet. Ber., 1900.

[197] Oui, Ann. de Gyn. et d'Obst., XXXVII, 1892.

[198] Burder, Centralbl. f. Kindernheilk, I, p. 70.

[199] Lyon, cité d'après Déchavanne, no 3.

[200] Hammerbacher, Arch. $f$. ges. Physiol, XXXIII, p. 228.

[201] Sonnenberg, cité d'après Karnik, no 3.

[202] ZeмuJc, l'Etude sur l'élimination de la solanine dans le lait des paches et la méthode biologique analytique, Thèse Brno, 1924.

\section{BIBLIOGRAPHIE ANALYTIQUE}

\section{LES LIVRES}

Genin (Ch.) et Rox (H.). - Etude sur la production et la vente du lait en Suisse, Bureau de la Gazette agricole, Lyon, 1925.

Cette intéressante brochure rassemble des notes sur un voyage d'études laitières en Suisse organisée sous les auspices de la Confédération générale des producteurs de lait, et le Secrétariat central du lait du Sud-Est.

Ce voyage eut lieu du 24 au 30 août 1925. Il avait pour but d'étudier les progrès obtenus en Suisse par l'organisation agricole et laitière.

Successivement dans leur brochure, les auteurs étudient le fonctionnement de l'Union suisse des paysans à Brugg, l'Union centrale des producteurs suisses de lait. Ils nous parlent de l'Ecole de laiterie de la Rutti, de l'Institut agricole du canton de Fribourg à Grangeneuve, des Laiteries réunies de Genève, de l'Etable coopératipe de Satigny et ils finissent par la description de l'usine édifiée à Rumilly par la Compagnie générale du lait.

Les conclusions de eette étude demandent à être reproduites in extenso :

" Que faut-rl retenir de Ce voyage d'Études? - L'agriculture suisse traverse en ce moment une période de prospérité remarquable; lorsque l'on parcourt les campagnes suisses, on a l'impression que tous les 